\title{
The study of mathematical models of the linear theory of elasticity by presenting the fundamental solution in harmonic potentials
}

\author{
Pabyrivskyi V. V., Pabyrivska N. V., Pukach P. Ya. \\ Lviv Polytechnic National University, \\ 12 S. Bandera Str., 79013, Lviv, Ukraine
}

(Received 28 January 2020; Revised 27 May 2020; Accepted 30 May 2020)

\begin{abstract}
In this paper, the approaches to the study of mathematical models of the theory of linear elasticity are developed. The general formulation of the 3-dimensional problem based on the representation of the fundamental solution in the form of V.P. Revenko in terms of spatial harmonic functions is considered. The formulation in the harmonic potentials of the 3-dimensional problem of elasticity in a cylindrical coordinate system for bodies bounded by the canonical surface is done. The boundary-value problems of pure rotation and circular symmetry in harmonic potentials are formulated. The mentioned approaches make it possible to obtain analytical solutions to these problems and are the theoretical basis for calculating the strength parameters of technical systems in a way of analysis of their mathematical models.
\end{abstract}

Keywords: elasticity theory, problem of torsion, fundamental solution, harmonic potentials, deformation tensor, stress tensor.

2010 MSC: 70C20, 74B05, 31B05

DOI: $10.23939 / \mathrm{mmc} 2020.02 .259$

\section{Introduction}

One of the main scientific directions of the study of boundary-value problems in deformable solid mechanics is the spatial problems of the theory of elasticity. For general and applied research, the relevance of this topic is determined by the fact that almost always the stress-strain state of a solid is of a 3-dimensional nature.

There are several methods for constructing analytical solutions to the spatial problems of static elasticity theory, one of which is based on the representation of homogeneous equations of equilibrium in terms of displacements using harmonic and biharmonic functions. These representations of the solutions have been considered in the classical works of such scientists as M. Boussinesq, W. Kelvin, P. Tait, B. G. Galerkin, and described in the monograph by S. P. Timoshenko [1]. The general solution based on the scalar and vector spatial harmonic functions has been for the first time proposed by P.F. Papkovich [2] and by G. Neiber [3]. Recent studies of the abovementioned issues have been published in the scientific papers of V.P. Revenko [4-6], where the general solution of homogeneous equations of equilibrium in terms of displacements was presented with the help of only three spatial harmonic functions and the volumetric expansion is expressed in terms of one of the above-mentioned functions. Relevant results are presented in Section 3 of this article. This solution representation has become the basis for formulating the boundary axisymmetric problems of elasticity theory in the harmonic potentials mentioned above, namely, the problems of pure rotation and circular symmetry for isotropic elastic bodies bounded by a canonical surface.

Note that the problems of research of spatial mathematical models of static theory of elasticity and related problems are considered, in particular, in the known monographs $[7,8]$. Problems solving of dynamics and strength of machines, elements of technological equipment implies the study of mathematical models described by dynamic boundary-value problems of the theory of elasticity $[9,10]$. At present, numerical [11,12], qualitative $[13,14]$ and analytical $[15,16]$ approaches to constructing solutions of boundary-value problems of dynamic and static theory of elasticity have been developed. 
Static problem analysis methods allow us to evaluate, predict, and optimize the structural strength parameters. The approaches developed in this paper to the construction of analytical solutions to the boundary-value problems of the axisymmetric theory of elasticity can be effectively used to analyze and synthesize the parameters of drilling equipment [17].

\section{Formulation of the problem of linear elasticity theory for the body of rotation}

We consider an isotropic elastic body $X$ in a 3-dimensional space, which is bounded by the surface $\partial X$ and whose surface is described by a canonical curve $f(r, \varphi, z)=0$ (Fig. 1).

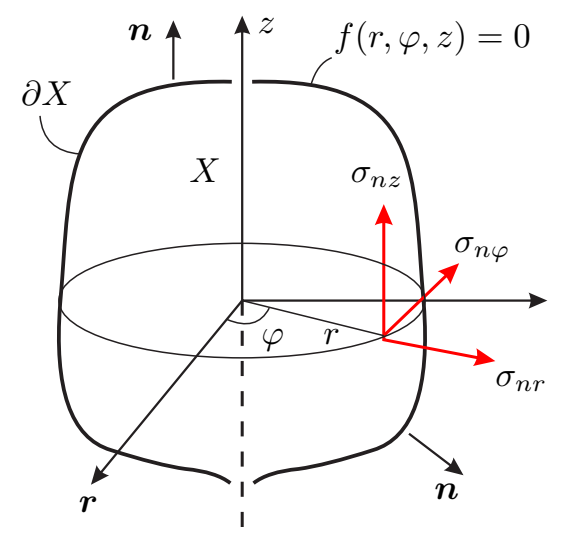

Fig. 1. Isotropic elastic body bounded by a canonical surface

The body is under the action of the stationary force load applied to the lateral surface of the body $\partial X$. The general equation of an elastic isotropic body equilibrium in the absence of bulk forces in a cylindrical coordinate system $(r, \varphi, z)$ has the form [1]

$$
\left\{\begin{array}{l}
\frac{\partial \sigma_{r}}{\partial r}+\frac{1}{r} \frac{\partial \sigma_{\varphi}}{\partial \varphi}+\frac{\partial \sigma_{r z}}{\partial z}+\frac{\sigma_{r}-\sigma_{\varphi}}{r}=0 \\
\frac{\partial \sigma_{r z}}{\partial r}+\frac{1}{r} \frac{\partial \sigma_{\varphi z}}{\partial \varphi}+\frac{\partial \sigma_{z}}{\partial z}+\frac{\sigma_{r z}}{r}=0 \\
\frac{\partial \sigma_{r \varphi}}{\partial r}+\frac{1}{r} \frac{\partial \sigma_{\varphi}}{\partial \varphi}+\frac{\partial \sigma_{\varphi z}}{\partial z}+\frac{2 \sigma_{r \varphi}}{r}=0
\end{array}\right.
$$

where $\sigma_{r}, \sigma_{\varphi}, \sigma_{z}, \sigma_{r \varphi}, \sigma_{r z}, \sigma_{z \varphi}$ are components of the stress tensor $\hat{\sigma}$, which satisfies the boundary conditions

$$
\left.\boldsymbol{\sigma}_{n} \equiv(\boldsymbol{n} \cdot \hat{\sigma})\right|_{\partial X}=\boldsymbol{\sigma}_{n}^{+}
$$

The stress tensor $\hat{\sigma}$ can be presented in terms of the deformation tensor as follows:

$$
\begin{gathered}
\sigma_{r}=\lambda \vartheta+2 \mu \varepsilon_{r}, \quad \sigma_{\varphi}=\lambda \vartheta+2 \mu \varepsilon_{\varphi}, \quad \sigma_{z}=\lambda \vartheta+2 \mu \varepsilon_{z}, \\
\sigma_{r \varphi}=\mu \varepsilon_{r \varphi}, \quad \sigma_{r z}=\mu \varepsilon_{r z}, \quad \sigma_{z \varphi}=\mu \varepsilon_{z \varphi},
\end{gathered}
$$

where $\varepsilon_{r}, \varepsilon_{\varphi}, \varepsilon_{z}, \varepsilon_{r \varphi}, \varepsilon_{r z}, \varepsilon_{\varphi z}$ are components of the deformation tensor $\hat{\varepsilon}$. These components of the stress tensor are determined in terms of the displacement vector $\boldsymbol{u}\left(u_{r}, u_{\varphi}, u_{z}\right)$ by the following relations:

$$
\begin{gathered}
\varepsilon_{r}=\frac{\partial u_{r}}{\partial r}, \quad \varepsilon_{\varphi}=\frac{u_{r}}{r}+\frac{1}{r} \frac{\partial u_{\varphi}}{\partial \varphi}, \quad \varepsilon_{z}=\frac{\partial u_{z}}{\partial z} \\
\varepsilon_{r \varphi}=\frac{1}{r} \frac{\partial u_{r}}{\partial \varphi}+\frac{\partial u_{\varphi}}{\partial r}-\frac{u_{\varphi}}{r}, \quad \varepsilon_{r z}=\frac{\partial u_{r}}{\partial z}+\frac{\partial u_{z}}{\partial r}, \quad \varepsilon_{\varphi z}=\frac{\partial u_{\varphi}}{\partial z}+\frac{1}{r} \frac{\partial u_{z}}{\partial \varphi} .
\end{gathered}
$$

We note that the volumetric expansion of the deformation tensor $\vartheta$ has the form

$$
\vartheta \equiv \varepsilon_{r}+\varepsilon_{\varphi}+\varepsilon_{z}=\frac{\partial u_{r}}{\partial r}+\frac{u_{r}}{r}+\frac{1}{r} \frac{\partial u_{\varphi}}{\partial \varphi}+\frac{\partial u_{z}}{\partial z}
$$

Here $\mu, \lambda$ are the Lamé parameters; $\boldsymbol{\sigma}_{n}(r, \varphi, z)$ is the vector of stresses on the surface $\partial X ; \boldsymbol{\sigma}_{n}^{+}(r, \varphi, z)$ is a given vector of surface efforts. The external normal vector $\boldsymbol{n}$ on the surface $\partial X$ is presented as follows:

$$
\boldsymbol{n} \equiv n_{r} \boldsymbol{e}_{\boldsymbol{r}}+n_{\varphi} \boldsymbol{e}_{\varphi}+n_{z} \boldsymbol{e}_{\boldsymbol{z}}=\frac{\boldsymbol{\nabla} f}{|\boldsymbol{\nabla} f|}
$$

where $\boldsymbol{\nabla}=\frac{\partial}{\partial r} \boldsymbol{e}_{\boldsymbol{r}}+\frac{1}{r} \frac{\partial}{\partial \varphi} \boldsymbol{e}_{\boldsymbol{\varphi}}+\frac{\partial}{\partial z} \boldsymbol{e}_{\boldsymbol{z}}$ is a Hamilton operator.

Mathematical Modeling and Computing, Vol.7, No. 2, pp. 259-268 (2020) 


\section{General formulation of the boundary-value problem of elasticity theory in harmonic potentials}

To construct a solution to the boundary-value problem of linear elasticity theory (1), (2), we consider the representation of the displacement vector $\boldsymbol{u}$ in the Revenko form [4] in terms of spatial independent scalar harmonic functions $R(r, \varphi, z), \Psi(r, \varphi, z), Q(r, \varphi, z)$ in the form:

$$
\boldsymbol{u}=z \operatorname{grad} R-(3-4 \nu) R \boldsymbol{e}_{\boldsymbol{z}}+\operatorname{grad} \Psi+\operatorname{rot} Q \boldsymbol{e}_{\boldsymbol{z}},
$$

which is the general solution to the equilibrium equation in displacements in the absence of bulk forces

$$
\mu \Delta \boldsymbol{u}+(\lambda+\mu) \boldsymbol{\nabla}(\boldsymbol{\nabla} \cdot \boldsymbol{u})=0 .
$$

Consider this representation in a cylindrical coordinate system $(r, \varphi, z)$. Since

$$
\begin{aligned}
& \operatorname{grad} R=\boldsymbol{\nabla} \cdot R=\frac{\partial R}{\partial r} \boldsymbol{e}_{\boldsymbol{r}}+\frac{1}{r} \frac{\partial R}{\partial \varphi} \boldsymbol{e}_{\boldsymbol{\varphi}}+\frac{\partial R}{\partial z} \boldsymbol{e}_{\boldsymbol{z}} \\
& \operatorname{grad} \Psi=\boldsymbol{\nabla} \cdot \Psi=\frac{\partial \Psi}{\partial r} \boldsymbol{e}_{\boldsymbol{r}}+\frac{1}{r} \frac{\partial \Psi}{\partial \varphi} \boldsymbol{e}_{\boldsymbol{\varphi}}+\frac{\partial \Psi}{\partial z} \boldsymbol{e}_{\boldsymbol{z}} \\
& \operatorname{rot}\left(Q \boldsymbol{e}_{\boldsymbol{z}}\right)=\boldsymbol{\nabla} \times\left(Q \boldsymbol{e}_{\boldsymbol{z}}\right)=\frac{1}{r} \frac{\partial Q}{\partial \varphi} \boldsymbol{e}_{\boldsymbol{r}}-\frac{\partial Q}{\partial r} \boldsymbol{e}_{\boldsymbol{\varphi}}
\end{aligned}
$$

$\Delta \equiv \boldsymbol{\nabla} \cdot \boldsymbol{\nabla}=\frac{\partial^{2}}{\partial r^{2}}+\frac{1}{r} \frac{\partial}{\partial r}+\frac{1}{r^{2}} \frac{\partial^{2}}{\partial \varphi^{2}}+\frac{\partial^{2}}{\partial z^{2}}$ is a Laplace operator, then the representation (3) takes the form

$$
\begin{aligned}
\boldsymbol{u} & =u_{r} \boldsymbol{e}_{\boldsymbol{r}}+u_{\varphi} \boldsymbol{e}_{\boldsymbol{\varphi}}+u_{z} \boldsymbol{e}_{\boldsymbol{z}} \\
& =\left(z \frac{\partial R}{\partial r}+\frac{\partial \Psi}{\partial r}+\frac{1}{r} \frac{\partial Q}{\partial \varphi}\right) \boldsymbol{e}_{\boldsymbol{r}}+\left(\frac{z}{r} \frac{\partial R}{\partial \varphi}+\frac{1}{r} \frac{\partial \Psi}{\partial \varphi}-\frac{\partial Q}{\partial r}\right) \boldsymbol{e}_{\boldsymbol{\varphi}}+\left(z \frac{\partial R}{\partial z}-(3-4 \nu) R+\frac{\partial \Psi}{\partial z}\right) \boldsymbol{e}_{\boldsymbol{z}}
\end{aligned}
$$

Accordingly, the components of the displacement vector $\boldsymbol{u}$ will have the following form:

$$
\begin{aligned}
& u_{r}=\frac{\partial \Psi}{\partial r}+\frac{1}{r} \frac{\partial Q}{\partial \varphi}+z \frac{\partial R}{\partial r}, \\
& u_{\varphi}=\frac{1}{r} \frac{\partial \Psi}{\partial \varphi}-\frac{\partial Q}{\partial r}+\frac{z}{r} \frac{\partial R}{\partial \varphi}, \\
& u_{z}=\frac{\partial \Psi}{\partial z}+z \frac{\partial R}{\partial z}-(3-4 \nu) R .
\end{aligned}
$$

On the basis of the relations (5)-(7), we present the components of the strain tensor $\hat{\varepsilon}$ :

$$
\begin{gathered}
\varepsilon_{r}=\frac{\partial u_{r}}{\partial r}=\frac{\partial^{2} \Psi}{\partial r^{2}}-\frac{1}{r^{2}} \frac{\partial Q}{\partial \varphi}+\frac{1}{r} \frac{\partial^{2} Q}{\partial \varphi \partial r}+z \frac{\partial^{2} R}{\partial r^{2}}, \\
\varepsilon_{\varphi}=\frac{u_{r}}{r}+\frac{1}{r} \frac{\partial u_{\varphi}}{\partial \varphi}=\frac{1}{r} \frac{\partial \Psi}{\partial r}+\frac{1}{r^{2}} \frac{\partial^{2} \Psi}{\partial \varphi^{2}}+\frac{1}{r^{2}} \frac{\partial Q}{\partial \varphi}-\frac{1}{r} \frac{\partial^{2} Q}{\partial r \partial \varphi}+\frac{z}{r} \frac{\partial R}{\partial r}+\frac{z}{r^{2}} \frac{\partial^{2} R}{\partial \varphi^{2}}, \\
\varepsilon_{z}=\frac{\partial u_{z}}{\partial z}=\frac{\partial^{2} \Psi}{\partial z^{2}}+z \frac{\partial^{2} R}{\partial z^{2}}+2(2 \nu-1) \frac{\partial R}{\partial z}, \\
\varepsilon_{r \varphi}=\frac{1}{r} \frac{\partial u_{r}}{\partial \varphi}+\frac{\partial u_{\varphi}}{\partial r}-\frac{u_{\varphi}}{r}=\frac{2}{r} \frac{\partial^{2} \Psi}{\partial r \partial \varphi}-\frac{2}{r^{2}} \frac{\partial \Psi}{\partial \varphi}+\frac{1}{r^{2}} \frac{\partial^{2} Q}{\partial \varphi^{2}}-\frac{\partial^{2} Q}{\partial r^{2}}+\frac{1}{r} \frac{\partial Q}{\partial r}+\frac{2 z}{r} \frac{\partial^{2} R}{\partial r \partial \varphi}-\frac{2 z}{r^{2}} \frac{\partial R}{\partial \varphi}, \\
\varepsilon_{r z}=\frac{\partial u_{r}}{\partial z}+\frac{\partial u_{z}}{\partial r}=2 \frac{\partial^{2} \Psi}{\partial r \partial z}+\frac{1}{r} \frac{\partial^{2} Q}{\partial \varphi \partial z}+2 z \frac{\partial^{2} R}{\partial z \partial r}+2(2 \nu-1) \frac{\partial R}{\partial r}, \\
\varepsilon_{\varphi z}=\frac{\partial u_{\varphi}}{\partial z}+\frac{1}{r} \frac{\partial u_{z}}{\partial \varphi}=\frac{2}{r} \frac{\partial^{2} \Psi}{\partial \varphi \partial z}-\frac{\partial^{2} Q}{\partial r \partial z}+\frac{2 z}{r} \frac{\partial^{2} R}{\partial z \partial \varphi}+(2 \nu-1) \frac{2}{r} \frac{\partial R}{\partial \varphi} .
\end{gathered}
$$

Mathematical Modeling and Computing, Vol. 7, No. 2, pp. 259-268 (2020) 
Then the volumetric expansion of the strain tensor $\vartheta$ takes the form:

$$
\begin{aligned}
\vartheta= & \varepsilon_{r}+\varepsilon_{\varphi}+\varepsilon_{z} \\
= & \frac{\partial^{2} \Psi}{\partial r^{2}}-\frac{1}{r^{2}} \frac{\partial Q}{\partial \varphi}+\frac{1}{r} \frac{\partial^{2} Q}{\partial r \partial \varphi}+z \frac{\partial^{2} R}{\partial r^{2}}+\frac{1}{r} \frac{\partial \Psi}{\partial r}+\frac{1}{r^{2}} \frac{\partial Q}{\partial \varphi}+\frac{z}{r} \frac{\partial R}{\partial r} \\
& +\frac{1}{r}\left[\frac{1}{r} \frac{\partial^{2} \Psi}{\partial \varphi^{2}}-\frac{\partial^{2} Q}{\partial r \partial \varphi}+z \frac{\partial^{2} R}{\partial \varphi^{2}}\right]+\frac{\partial^{2} \Psi}{\partial z^{2}}+z \frac{\partial^{2} R}{\partial z^{2}}+2(2 \nu-1) \frac{\partial R}{\partial z} .
\end{aligned}
$$

Since the functions $R(r, \varphi, z), \Psi(r, \varphi, z), Q(r, \varphi, z)$ are harmonic, i.e. $\Delta(R, \Psi, Q)=0$, then

$$
\vartheta=\Delta \Psi+z \Delta R-2(1-2 \nu) \frac{\partial R}{\partial z}=-2(1-2 \nu) \frac{\partial R}{\partial z} .
$$

This fact makes it possible to more easily obtain solutions to the boundary-value problems of linear theory of elasticity. Accordingly, the components of the stress tensor $\sigma_{r}, \sigma_{\varphi}, \sigma_{z}, \sigma_{r \varphi}, \sigma_{r z}, \sigma_{z \varphi}$ can be represented as follows:

$$
\begin{gathered}
\sigma_{r}=\lambda \vartheta+2 \mu \varepsilon_{r}=2 \mu\left[\frac{\partial^{2}(\Psi+\mathrm{zR})}{\partial r^{2}}+\frac{\partial}{\partial r}\left(\frac{1}{r} \frac{\partial Q}{\partial \varphi}\right)-2 \nu \frac{\partial R}{\partial z}\right] \\
\sigma_{\varphi}=\lambda \vartheta+2 \mu \varepsilon_{\varphi}=2 \mu\left[\frac{1}{r^{2}} \frac{\partial^{2}(\Psi+\mathrm{z} R)}{\partial \varphi^{2}}+\frac{1}{r} \frac{\partial(\Psi+z R)}{\partial r}-\frac{\partial}{\partial r}\left(\frac{1}{r} \frac{\partial Q}{\partial \varphi}\right)-2 \nu \frac{\partial R}{\partial z}\right], \\
\sigma_{z}=\lambda \vartheta+2 \mu \varepsilon_{z}=2 \mu\left[\frac{\partial^{2} \Psi}{\partial z^{2}}+\mathrm{z} \frac{\partial^{2} R}{\partial z^{2}}+2(\nu-1) \frac{\partial R}{\partial z}\right], \\
\sigma_{r \varphi}=\mu \varepsilon_{r \varphi}=\mu\left[2 \frac{\partial}{\partial r}\left(\frac{1}{r} \frac{\partial \Psi}{\partial \varphi}\right)+\frac{1}{r^{2}} \frac{\partial^{2} Q}{\partial \varphi^{2}}-\frac{\partial^{2} Q}{\partial r^{2}}+\frac{1}{r} \frac{\partial Q}{\partial r}+2 z \frac{\partial}{\partial r}\left(\frac{1}{r} \frac{\partial R}{\partial \varphi}\right)\right], \\
\sigma_{r z}=\mu \varepsilon_{r z}=\mu\left[2 \frac{\partial^{2} \Psi}{\partial r \partial z}+\frac{1}{r} \frac{\partial^{2} Q}{\partial \varphi \partial z}+2 z \frac{\partial^{2} R}{\partial z \partial r}+2(2 \nu-1) \frac{\partial R}{\partial r}\right] \\
\sigma_{z \varphi}=\mu \varepsilon_{z \varphi}=\mu\left[\frac{2}{r} \frac{\partial^{2} \Psi}{\partial \varphi \partial z}-\frac{\partial^{2} Q}{\partial r \partial z}+\frac{2 z}{r} \frac{\partial^{2} R}{\partial z \partial \varphi}+(2 \nu-1) \frac{2}{r} \frac{\partial R}{\partial \varphi}\right]
\end{gathered}
$$

It is easy to see that the representation of the stress tensor by the relation (8) satisfies identically the equilibrium equation (1).

Thus, using the fundamental solution representation of Revenko, the boundary-value problem of linear elasticity theory (1), (2) is reformulated to a boundary-value problem for spatial harmonic functions $R(r, \varphi, z), \Psi(r, \varphi, z), Q(r, \varphi, z)$ in the form $\Delta(R, \Psi, Q)=0$, which satisfy the corresponding boundary conditions on the surface $\partial X$ :

$$
\begin{aligned}
\left.\boldsymbol{\sigma}_{n} \equiv(\boldsymbol{n} \cdot \hat{\sigma})\right|_{\partial X}=\left(\sigma_{n r} \boldsymbol{e}_{\boldsymbol{r}}\right. & \left.+\sigma_{n \varphi} \boldsymbol{e}_{\boldsymbol{\varphi}}+\sigma_{n z} \boldsymbol{e}_{\boldsymbol{z}}\right)\left.\right|_{\partial X}=\left(\left(n_{r} \sigma_{r r}+n_{\varphi} \sigma_{\varphi r}+n_{z} \sigma_{z r}\right) \boldsymbol{e}_{\boldsymbol{r}}\right. \\
+ & \left.\left(n_{r} \sigma_{r \varphi}+n_{\varphi} \sigma_{\varphi \varphi}+n_{z} \sigma_{z \varphi}\right) \boldsymbol{e}_{\boldsymbol{\varphi}}+\left(n_{r} \sigma_{r z}+n_{\varphi} \sigma_{\varphi z}+n_{z} \sigma_{z z}\right) \boldsymbol{e}_{\boldsymbol{z}}\right)\left.\right|_{\partial X}=\boldsymbol{\sigma}_{n}^{+}
\end{aligned}
$$

Let us illustrate the application of the representation (3) in terms of the harmonic functions $R(r, \varphi, z)$, $\Psi(r, \varphi, z), Q(r, \varphi, z)$ to construct analytical solutions of the 3-dimensional rotation problem of the linear theory of elasticity. In this case, the following distribution is correct for the stress tensor components:

$$
\sigma_{r}=0, \quad \sigma_{\varphi}=0, \quad \sigma_{z}=0 .
$$

Based on this condition, the volumetric deformation of the strain tensor $\vartheta$ is zero and, accordingly, $R=0$. Then, according to the representation (8), the above-mentioned condition can be written in terms of the harmonic potentials $\Psi(r, \varphi, z), Q(r, \varphi, z)$ as follows:

$$
\sigma_{r}=\frac{\partial}{\partial r}\left(\frac{\partial \Psi}{\partial r}+\frac{1}{r} \frac{\partial Q}{\partial \varphi}\right)=0, \quad \sigma_{z}=\frac{\partial^{2} \Psi}{\partial z^{2}}=0, \quad \sigma_{\varphi}=\frac{1}{r} \frac{\partial}{\partial \varphi}\left(\frac{1}{r} \frac{\partial \Psi}{\partial \varphi}-\frac{\partial Q}{\partial r}\right)+\frac{1}{r}\left(\frac{\partial \Psi}{\partial r}+\frac{1}{r} \frac{\partial Q}{\partial \varphi}\right)=0 .
$$

Mathematical Modeling and Computing, Vol. 7, No. 2, pp. 259-268 (2020) 
From the first two relations we obtain the structure of the harmonic functions $\Psi(r, \varphi, z), Q(r, \varphi, z)$ in the form

$$
\begin{gathered}
\Psi(r, \varphi, z)=\Psi_{0}(r, \varphi)+z \Psi_{1}(r, \varphi), \\
Q(r, \varphi, z)=Q_{0}(r, z)+Q_{1}(r, \varphi)+z Q_{2}(r, \varphi),
\end{gathered}
$$

where $\Psi_{i}, Q_{i}(i=\overline{0,2})$ are unknown harmonic functions.

We apply this representation to the relations of the stress tensor (8). Note that the harmonic potentials $\Psi_{0}(r, \varphi)$ and $Q_{1}(r, \varphi)$ do not affect the structure of tangential stresses $\sigma_{r z}, \sigma_{z \varphi}$, which are components of the moment characteristics of external loads, so they can be set equal to zero. Thus, performing the transformation, we obtain the following form of the components of the stress tensor:

$$
\begin{gathered}
\sigma_{r}=\frac{\partial}{\partial r}\left(\frac{\partial \Psi_{1}}{\partial r}+\frac{1}{r} \frac{\partial Q_{2}}{\partial \varphi}\right)=0, \quad \sigma_{\varphi}=\frac{1}{r} \frac{\partial}{\partial \varphi}\left(\frac{1}{r} \frac{\partial \Psi_{1}}{\partial \varphi}-\frac{\partial Q_{2}}{\partial r}\right)+\frac{1}{r}\left(\frac{\partial \Psi_{1}}{\partial r}+\frac{1}{r} \frac{\partial Q_{2}}{\partial \varphi}\right)=0, \quad \sigma_{z}=0 \\
\sigma_{r \varphi}=\mu\left[\left(-\frac{\partial^{2} Q_{0}}{\partial r^{2}}+\frac{1}{r} \frac{\partial Q_{0}}{\partial r}\right)+2 z \frac{\partial}{\partial r}\left(\frac{1}{r} \frac{\partial \Psi_{1}}{\partial \varphi}-\frac{\partial Q_{2}}{\partial r}\right)\right], \quad \sigma_{r z}=\mu\left[\frac{\partial \Psi_{1}}{\partial r}+\left(\frac{\partial \Psi_{1}}{\partial r}+\frac{1}{r} \frac{\partial Q_{2}}{\partial \varphi}\right)\right], \\
\sigma_{z \varphi}=\mu\left[\frac{1}{r} \frac{\partial \Psi_{1}}{\partial \varphi}-\frac{\partial^{2} Q_{0}}{\partial r \partial z}+\left(\frac{1}{r} \frac{\partial \Psi_{1}}{\partial \varphi}-\frac{\partial Q_{2}}{\partial r}\right)\right] .
\end{gathered}
$$

Since the functions $\Psi_{1}, Q_{2}$ satisfy the Laplace equation $\Delta \equiv\left(\frac{1}{r} \frac{\partial}{\partial r}\left(r \frac{\partial}{\partial r}\right)+\frac{1}{r^{2}} \frac{\partial^{2}}{\partial \varphi^{2}}\right)\left(\Psi_{1}, Q_{2}\right)=0$, then we obtain, in particular, the following relations:

$$
\frac{\partial \Psi_{1}}{\partial r}=-\frac{1}{r} \frac{\partial Q_{2}}{\partial \varphi}, \quad \frac{1}{r} \frac{\partial \Psi_{1}}{\partial \varphi}=\frac{\partial Q_{2}}{\partial r}
$$

Based on these relations, we present the final version of the components of the stress tensor through two harmonic potentials $Q_{0}(r, z), \Psi_{1}(r, \varphi)$ in the form:

$$
\sigma_{r}=0, \quad \sigma_{\varphi}=0, \quad \sigma_{\mathrm{z}}=0,
$$

$\sigma_{r \varphi}=\mu\left[-\frac{\partial^{2} Q_{0}(r, z)}{\partial r^{2}}+\frac{1}{r} \frac{\partial Q_{0}(r, z)}{\partial r}\right], \quad \sigma_{r z}=\mu\left[\frac{\partial \Psi_{1}(r, \varphi)}{\partial r}\right], \quad \sigma_{z \varphi}=\mu\left[\frac{1}{r} \frac{\partial \Psi_{1}(r, \varphi)}{\partial \varphi}-\frac{\partial^{2} Q_{0}(r, z)}{\partial r \partial z}\right]$,

which form the structure of the vectors of the corresponding external loads on the body surface $\partial X$ :

$$
\begin{aligned}
\boldsymbol{\sigma}_{n}= & {\left[\left(n_{\varphi}\left(-\frac{\partial^{2} Q_{0}(r, z)}{\partial r^{2}}+\frac{1}{r} \frac{\partial Q_{0}(r, z)}{\partial r}\right)+n_{z}\left(\frac{\partial \Psi_{1}(r, \varphi)}{\partial r}\right)\right) \boldsymbol{e}_{\boldsymbol{r}}\right.} \\
& +\left(n_{r}\left(-\frac{\partial^{2} Q_{0}(r, z)}{\partial r^{2}}+\frac{1}{r} \frac{\partial Q_{0}(r, z)}{\partial r}\right)+n_{z}\left(\frac{1}{r} \frac{\partial \Psi_{1}(r, \varphi)}{\partial \varphi}-\frac{\partial^{2} Q_{0}(r, z)}{\partial r \partial z}\right)\right) \boldsymbol{e}_{\boldsymbol{\varphi}} \\
& \left.+\left(n_{\varphi}\left(-\frac{\partial^{2} Q_{0}(r, z)}{\partial r^{2}}+\frac{1}{r} \frac{\partial Q_{0}(r, z)}{\partial r}\right)+n_{z}\left(\frac{\partial \Psi_{1}(r, \varphi)}{\partial r}\right)\right) \boldsymbol{e}_{\boldsymbol{r}}\right]\left.\right|_{\partial X}=\boldsymbol{\sigma}_{n}^{+}
\end{aligned}
$$

Accordingly, the components of the displacement vector $\boldsymbol{u}=\left(u_{r}, u_{\varphi}, u_{z}\right)$ in terms of the specified potentials are given as follows:

$$
u_{r}=0, \quad u_{\varphi}=-\frac{\partial Q_{0}(r, z)}{\partial r}, \quad u_{z}=\Psi_{1}(r, \varphi)
$$

In [18], an example of using the developed methods for constructing a set of exact analytical solutions of boundary-value problems of spatial theory of elasticity is considered. The example is based on the representation of the general solution of the equation of the linear theory of elasticity in the form of Papkovich-Neuber through scalar and vector harmonic functions [2,3]. By generalizing the Cauchy-Riemann conditions, the displacement vector and the stress tensor are represented through 
scalar and vector holomorphic functions of two complex variables, which in turn are given in the form of polynomials of order $n$ by powers of complex variables $z_{1}$ and $z_{2}$. The corresponding boundary conditions for the basic solutions are formulated and the integral conditions of zero equality of the principal moment of the stress vector on the lateral surface of the body are concretized.

The mentioned boundary-value problem simulates the distribution of stresses and corresponding external loads on the lateral surface of the drill. As follows from the results of the study of the mathematical model of the linear theory of elasticity considered in this paper, the proposed approach allows us to construct and analyze the structure of external force load on the basis of the general solution in the form of Revenko. This can be used to synthesize the parameters of many mechanical systems.

\section{Formulation of axisymmetric problems of elasticity theory}

For the formulated boundary-value problem (1), (2), we consider the case of axisymmetric boundaryvalue problems. Let the $z$-axis coincide with the axis of symmetry of the rotating body (Fig. 1), which is under the action of forces symmetrical to the axis of rotation. Then the components of the displacement vector $u_{r}=u_{r}(r, z), u_{\varphi}=u_{\varphi}(r, z), u_{z}=u_{z}(r, z)$ in the direction of the axes $r, \varphi, z$ are independent of the coordinate $\varphi$.

Under such conditions, the components of the strain tensor $\hat{\varepsilon}$, due to the independence of the displacement vector $\boldsymbol{u}$ from the coordinate $\varphi$ have the form:

$$
\varepsilon_{r}=\frac{\partial u_{r}}{\partial r}, \quad \varepsilon_{\varphi}=\frac{u_{r}}{r}, \quad \varepsilon_{z}=\frac{\partial u_{z}}{\partial z}, \quad \varepsilon_{r \varphi}=\frac{\partial u_{\varphi}}{\partial r}-\frac{u_{\varphi}}{r}, \quad \varepsilon_{r z}=\frac{\partial u_{r}}{\partial z}+\frac{\partial u_{z}}{\partial r}, \quad \varepsilon_{\varphi z}=\frac{\partial u_{\varphi}}{\partial z} .
$$

According to the formulation of the axisymmetric boundary-value problem for the components of the displacement vector $\boldsymbol{u}$ in the form (5)-(7) the conditions are imposed $\frac{\partial \boldsymbol{u}}{\partial \varphi}=0$.

Given this condition, let us present the components of the elasticity tensor $\hat{\sigma}(8)$ in terms of the above-mentioned harmonic potentials:

$$
\begin{gathered}
\sigma_{r}=2 \mu\left[\frac{\partial^{2} \Psi}{\partial r^{2}}+z \frac{\partial^{2} R}{\partial r^{2}}-2 \nu \frac{\partial R}{\partial z}\right], \quad \sigma_{\varphi}=2 \mu\left[\frac{1}{r} \frac{\partial \Psi}{\partial r}+\frac{z}{r} \frac{\partial R}{\partial r}-2 \nu \frac{\partial R}{\partial z}\right], \\
\sigma_{z}=2 \mu\left[\frac{\partial^{2} \Psi}{\partial z^{2}}+z \frac{\partial^{2} R}{\partial z^{2}}-2(1-\nu) \frac{\partial R}{\partial z}\right], \\
\sigma_{r \varphi}=\mu \varepsilon_{r \varphi}=\mu\left[-\frac{\partial^{2} Q}{\partial r^{2}}+\frac{1}{r} \frac{\partial Q}{\partial r}\right], \quad \sigma_{r z}=\mu \varepsilon_{r z}=\mu\left[2 \frac{\partial^{2} \Psi}{\partial r \partial z}+2 z \frac{\partial^{2} R}{\partial z \partial r}-2(1-2 \nu) \frac{\partial R}{\partial r}\right], \\
\sigma_{z \varphi}=\mu \varepsilon_{z \varphi}=-\mu \frac{\partial^{2} Q}{\partial r \partial z} .
\end{gathered}
$$

Note that the linear invariant $I_{1}(\hat{\sigma})$ of the stress tensor $\hat{\sigma}$ is expressed only in terms of one harmonic function $R(r, z)$ and will have the form

$$
I_{1}(\hat{\sigma}) \equiv \sigma_{r}+\sigma_{\varphi}+\sigma_{z}=2 \mu\left(\Delta \Psi+z \Delta R-2(\nu+1) \frac{\partial R}{\partial z}\right)=-4 \mu(1+\nu) \frac{\partial R}{\partial z}=-2 E \frac{\partial R}{\partial z},
$$

where $E$ is the Young modulus.

Thus, the boundary-value problem on harmonic potentials $\Psi(r, \varphi, z), R(r, \varphi, z), Q(r, \varphi, z)$ in the axisymmetric formulation has the form $\Delta(R, \Psi, Q)=0$, moreover, the boundary conditions on the surface $\partial X$ have the form:

$$
\boldsymbol{\sigma}_{n}=\mu\left[\left(2 n_{r}\left(\frac{\partial^{2} \Psi}{\partial r^{2}}+z \frac{\partial^{2} R}{\partial r^{2}}-2 \nu \frac{\partial R}{\partial z}\right)+n_{\varphi}\left(-\frac{\partial^{2} Q}{\partial r^{2}}+\frac{1}{r} \frac{\partial Q}{\partial r}\right)\right.\right.
$$

Mathematical Modeling and Computing, Vol.7, No. 2, pp. 259-268 (2020) 


$$
\begin{gathered}
\left.+n_{z}\left(2 \frac{\partial^{2} \Psi}{\partial r \partial z}+2 z \frac{\partial^{2} R}{\partial z \partial r}-2(1-2 \nu) \frac{\partial R}{\partial r}\right)\right) \boldsymbol{e}_{\boldsymbol{r}} \\
+\left(n_{r}\left(-\frac{\partial^{2} Q}{\partial r^{2}}+\frac{1}{r} \frac{\partial Q}{\partial r}\right)+2 n_{\varphi}\left(\frac{1}{r} \frac{\partial \Psi}{\partial r}+\frac{z}{r} \frac{\partial R}{\partial r}-2 \nu \frac{\partial R}{\partial z}\right)+n_{z}\left(-\frac{\partial^{2} Q}{\partial r \partial z}\right)\right) \boldsymbol{e}_{\boldsymbol{\varphi}} \\
+\left(n_{r}\left(2 \frac{\partial^{2} \Psi}{\partial r \partial z}+2 z \frac{\partial^{2} R}{\partial z \partial r}-2(1-2 \nu) \frac{\partial R}{\partial r}\right)+n_{\varphi}\left(-\frac{\partial^{2} Q}{\partial r \partial z}\right)\right. \\
\left.\left.+2 n_{z}\left(\frac{\partial^{2} \Psi}{\partial z^{2}}+z \frac{\partial^{2} R}{\partial z^{2}}-2(1-\nu) \frac{\partial R}{\partial z}\right) \boldsymbol{e}_{\boldsymbol{z}}\right)\right]\left.\right|_{\partial X}=\boldsymbol{\sigma}_{n}^{+} .
\end{gathered}
$$

The general axisymmetric stress state of the body of rotation, which is symmetric to the $z$ axis, can be considered as the superposition of two independent elastic states, namely: pure rotation and circular symmetry. The first of the above-mentioned elastic states is described by equation of equilibrium (4) under conditions [1]:

$$
u_{r}=u_{z}=0
$$

Based on this condition, the components of the strain tensor $\hat{\varepsilon}$ and stresses $\hat{\sigma}$ will have the forms:

$$
\begin{gathered}
\varepsilon_{r}=\varepsilon_{\varphi}=\varepsilon_{z}=\varepsilon_{r z}=0, \quad \varepsilon_{r \varphi}=\frac{\partial u_{\varphi}}{\partial r}-\frac{u_{\varphi}}{r}, \quad \varepsilon_{\varphi z}=\frac{\partial u_{\varphi}}{\partial z} \\
\sigma_{r}=\sigma_{\varphi}=\sigma_{z}=\sigma_{r z}=0, \quad \sigma_{r \varphi}=\mu \varepsilon_{r \varphi}=\mu\left(\frac{\partial u_{\varphi}}{\partial r}-\frac{u_{\varphi}}{r}\right), \quad \sigma_{z \varphi}=\mu \varepsilon_{z \varphi}=\mu \frac{\partial u_{\varphi}}{\partial z} .
\end{gathered}
$$

Under the condition of rotation (9), the nonzero component of the displacement vector in the representation (3) will be expressed only in terms of a single harmonic potential $Q(r, z)$ in the form

$$
u_{\varphi}=-\frac{\partial Q(r, z)}{\partial r} .
$$

Accordingly, the volume expansion $\vartheta$ of the strain tensor $\hat{\varepsilon}$ will also be zero, i.e.

$$
\vartheta=\varepsilon_{r}+\varepsilon_{\varphi}+\varepsilon_{z}=\frac{\partial R(r, \varphi, z)}{\partial z}=0 .
$$

Based on this relation, we obtain the expressions for nonzero components of the stress tensor $\sigma_{r \varphi}, \sigma_{z \varphi}$ in terms of the harmonic potential $Q(r, z)$ :

$$
\sigma_{r \varphi}=\mu \varepsilon_{r \varphi}=\mu\left[-\frac{\partial^{2} Q}{\partial r^{2}}+\frac{1}{r} \frac{\partial Q}{\partial r}\right], \quad \sigma_{z \varphi}=\mu \varepsilon_{\varphi z}=-\mu \frac{\partial^{2} Q}{\partial r \partial z} .
$$

Thus, using the representation of the general solution in the form of Revenko (3), the boundaryvalue problem of rotation of the linear theory of elasticity is reformulated into the boundary-value problem of finding the harmonic potential $Q(r, z)$, which satisfies the corresponding boundary conditions on the body surface $\partial X$ :

$$
\begin{aligned}
\boldsymbol{\sigma}_{n} & \left.\equiv(\boldsymbol{n} \cdot \hat{\sigma})\right|_{\partial X} \\
& =\left.\mu\left\{n_{\varphi}\left[\left(\frac{1}{r} \frac{\partial Q}{\partial r}-\frac{\partial^{2} Q}{\partial r^{2}}\right)\right] \boldsymbol{e}_{\boldsymbol{r}}+\left(n_{r}\left[\left(\frac{1}{r} \frac{\partial Q}{\partial r}-\frac{\partial^{2} Q}{\partial r^{2}}\right)\right]+n_{z}\left[-\frac{\partial^{2} Q}{\partial r \partial z}\right]\right) \boldsymbol{e}_{\boldsymbol{\varphi}}+n_{\varphi}\left[-\frac{\partial^{2} Q}{\partial r \partial z}\right] \boldsymbol{e}_{\boldsymbol{z}}\right\}\right|_{\partial X} \\
& =\boldsymbol{\sigma}_{n}^{+}
\end{aligned}
$$

Consider another axisymmetric stress state of the body of rotation, namely the circular symmetry, which is described by the equation equilibrium (4) under conditions [1]:

$$
u_{\varphi}=0 .
$$


Under these conditions, the representation of the displacement vector (3) will have the form:

$$
u_{r}=\frac{\partial \Psi}{\partial r}+z \frac{\partial R}{\partial r}, \quad u_{\varphi}=-\frac{\partial Q}{\partial r}=0, \quad u_{z}=\frac{\partial \Psi}{\partial z}+z \frac{\partial R}{\partial z}-(3-4 \nu) R .
$$

Accordingly, the components of the strain tensor $\hat{\varepsilon}$ and the volumetric expansion $\vartheta$ will be written as follows:

$$
\begin{gathered}
\varepsilon_{r}=\frac{\partial^{2} \Psi}{\partial r^{2}}+z \frac{\partial^{2} R}{\partial r^{2}}, \quad \varepsilon_{\varphi}=\frac{1}{r} \frac{\partial \Psi}{\partial r}+\frac{z}{r} \frac{\partial R}{\partial r}, \quad \varepsilon_{z}=\frac{\partial^{2} \Psi}{\partial z^{2}}+z \frac{\partial^{2} R}{\partial z^{2}}-2(1-2 \nu) \frac{\partial R}{\partial z} \\
\varepsilon_{r z}=2 \frac{\partial}{\partial r}\left(\frac{\partial \Psi}{\partial z}+z \frac{\partial R}{\partial z}-(1-2 \nu) R\right), \quad \varepsilon_{r \varphi}=0, \quad \varepsilon_{z \varphi}=0, \quad \vartheta=\varepsilon_{r}+\varepsilon_{\varphi}+\varepsilon_{z}=-2(1-2 \nu) \frac{\partial R}{\partial z} .
\end{gathered}
$$

Therefore, the components of the stress tensor $\hat{\sigma}(8)$ are given only through two harmonic functions $R(r, z), \Psi(r, z)$ :

$$
\begin{gathered}
\sigma_{r}=2 \mu\left(\frac{\partial^{2}(\Psi+z R)}{\partial r^{2}}-2 \nu \frac{\partial R}{\partial z}\right), \quad \sigma_{z}=2 \mu \frac{\partial}{\partial z}\left(\frac{\partial(\Psi+z R)}{\partial z}-2(2-\nu) R\right) \\
\sigma_{\varphi}=2 \mu\left(\frac{1}{r} \frac{\partial(\Psi+z R)}{\partial r}-2 \nu \frac{\partial R}{\partial z}\right), \quad \sigma_{r z}=2 \mu \frac{\partial}{\partial r}\left(\frac{\partial(\Psi+z R)}{\partial z}-2(1-\nu) R\right), \quad \sigma_{r \varphi}=0, \quad \sigma_{z \varphi}=0 .
\end{gathered}
$$

Thus, the boundary-value problem of circular symmetry is reduced to finding the harmonic potentials $R(r, z), \Psi(r, z)$, which satisfy the boundary conditions on the body surface $\partial X$ :

$$
\begin{aligned}
\boldsymbol{\sigma}_{n}= & 2 \mu\left[\left(n_{r}\left(\frac{\partial^{2}(\Psi+z R)}{\partial r^{2}}-2 \nu \frac{\partial R}{\partial z}\right)+n_{z} \frac{\partial}{\partial r}\left(\frac{\partial(\Psi+z R)}{\partial z}-2(1-\nu) R\right)\right) \boldsymbol{e}_{\boldsymbol{r}}\right. \\
& +\left(n_{\varphi}\left(\frac{1}{r} \frac{\partial(\Psi+z R)}{\partial r}-2 \nu \frac{\partial R}{\partial z}\right)\right) \boldsymbol{e}_{\boldsymbol{\varphi}} \\
+ & \left.\left(\left(n_{r} \frac{\partial}{\partial r}\left(\frac{\partial(\Psi+z R)}{\partial z}-2(1-\nu) R\right)+n_{z} \frac{\partial}{\partial z}\left(\frac{\partial(\Psi+z R)}{\partial z}-2(2-\nu) R\right)\right) \boldsymbol{e}_{\boldsymbol{z}}\right]\right|_{\partial X}=\boldsymbol{\sigma}_{n}^{+}
\end{aligned}
$$

\section{Conclusions}

In this paper, the approaches to the study of static mathematical models of linear elasticity theory are developed. The boundary-value problem of the linear theory of elasticity for an isotropic body of rotation in 3-dimensional formulation is considered. Based on the presentation of a general solution of equilibrium equations in terms of spatial harmonic functions in a cylindrical coordinate system in the form of Revenko, the boundary-value problem of theory of elasticity is reformulated to the boundary-value problem for harmonic functions for the mentioned body.

The problem of finding the exact analytical solutions considered in the work is an actual scientific problem. In comparison with the numerical approaches that have been used and are still being used for this class of mathematical models, the possibility to obtain the exact analytical solutions allows us to describe the patterns of behavior of the mechanical systems discussed in this article in the best way.

Using the method developed in this article has made it possible to obtain the expressions of the components of stress and strain tensors in the three-dimensional formulation in terms of harmonic potentials. As an example, the class of axisymmetric problems is considered, namely: problems of pure rotation and axial symmetry; formulate appropriate boundary conditions for harmonic functions for the specified problems; offer a practical approach to the construction and analysis of the structure of external force load in the problems of synthesis and optimization of parameters of technological systems. 
The results obtained and the methods developed in this paper can be used in engineering practice, in particular, in the problems of optimization of the parameters of technological systems and the calculation of the strength of structures.

[1] Timoshenko S. P., Gudyer D. Teoriya uprugosti. Moscow, Nauka (1975), (in Russian).

[2] Papkovich P. F. Teoriya uprugosti. Moscow, Oborongiz (1939), (in Russian).

[3] Neyber G. Kontsentratsiya napryazheniy. Moscow, Gostekhizdat (1947), (in Russian).

[4] Revenko V.P. Solving the three-dimensional equations of the linear theory of elasticity. Int. Appl. Mech. 45 (7), 730-741 (2009).

[5] Revenko V.P. Construction of the general solution to the Lame three-dimensions equations of elasticity theory. Matematychnyi visnyk Naukovoho tovarystva im. Shevchenka. 2, 185-198 (2005), (in Ukrainian).

[6] Revenko V. P. Solution of 3d boundary value problem of elasticity theory for bodie of rotation. Prykladni problemy mekhaniky i matematyky. 12, 130-136 (2014), (in Ukrainian).

[7] Podilchuk Yu. N. Prostranstvennyye zadachi teorii uprugosti i plastichnosti (pod obshchey redaktsiyey A. N. Guz'). Krayevyye zadachi statiki uprugogo tela. Kyiv, Naukova Dumka (1984), (in Russian).

[8] Mors P. M., Feshbakh H. Methods of Theoretical Physics. Part 2. McGraw-Hill Science/Engineering/Math (1953).

[9] Pukach P. Ya., Kuzio I. V., Nytrebych Z. M., Ilkiv V. S. Analytical methods for determining the effect of the dynamic process on the nonlinear flexural vibrations and the strength of compressed shaft. Naukovyi Visnyk Natsionalnoho Hirnychoho Universytetu. 5, 69-76 (2017).

[10] Pukach P. Ya., Kuzio I. V., NytrebychZ.M., Ilkiv V.S. Asymptotic method for investigating resonant regimes of nonlinear bending vibrations of elastic shaft. Naukovyi Visnyk Natsionalnoho Hirnychoho Universytetu. 1, 68-73 (2018).

[11] Labibov R. R., Chernyakov Y. A., Sheveleva A.E., Shevchenko A. G. Strips of localization of plastic deformation. Archive of Applied Mechanics. 88 (12), 2221-2230 (2018).

[12] Sheveleva A., Lapusta Y., Loboda V. Opening and contact zones of an interface crack in a piezoelectric bimaterial under combined compressive-shear loading. Mechanics Research Communications. 63, 6-12 (2015).

[13] Pukach P. Ya. Investigation of bending vibrations in Voigt-Kelvin bars with regard for nonlinear resistance forces. J. Math. Sci. 215 (1), 71-78 (2016).

[14] Pukach P. Ya. Qualitative Methods for the Investigation of a Mathematical Model of Nonlinear Vibrations of a Conveyer Belt. J. Math. Sci. 198 (1), 31-38 (2014).

[15] Polozhiy G. N. Teoriya i primeneniye $p$-analiticheskikh i $(p, q)$-analiticheskikh funktsiy. Moscow, Nauka (1973), (in Russian).

[16] Burak Ya. I., Pabyrivskyy V.V. Pobudova rozviazkiv prostorovykh zadach teorii pruzhnosti z vykorystanniam metodu holomorfnykh funktsii dvokh kompleksnykh zminnykh. Lviv, Rastr-7 (2014), (in Ukrainian).

[17] Bondarenko V. I., Samusya V. I., Smolanov S. N. Mobile lifting units for wrecking works in pit shafts. Gornyi Zhurnal. 5, 99-100 (2005).

[18] Pabyrivskyi V. V., Kuzio I. V., Pabyrivska N. V., Pukach P. Ya. Two-dimensional elastic theory methods for describing the stress state and the modes of elastic boring. Naukovyi Visnyk Natsionalnoho Hirnychoho Universytetu. 1, 46-51 (2020). 


\title{
Дослідження математичних моделей лінійної теорії пружності шляхом подання фундаментального розв'язку в гармонійних потенціалах
}

\author{
Пабирівський В. В., Пабирівська Н. В., Пукач П. Я. \\ Національний університет "Лъвівсъка політехніка", \\ вул. С. Бандери, 12, 79013, Львів, Україна
}

\begin{abstract}
У роботі розвинуто підходи до дослідження математичних моделей лінійної теорії пружності. Розглядається загальне формулювання задачі на основі подання фундаментального розв'язку у формі Ревенка В. П. через просторові гармонійні функції. Здійснено постановку в гармонічних потенціалах тривимірної задачі пружності в циліндричній системі координат для тіл, обмежених канонічною поверхнею. Сформульовано крайові задачі чистого кручення та кругової симетрії в гармонічних потенціалах. Вказані підходи дають змогу отримати аналітичні розв'язки цих задач та $€$ теоретичним підгрунтям розрахунку параметрів міцності технічних систем шляхом аналізу їх математичних моделей.
\end{abstract}

Ключові слова: теорія пружності, задача кручення, фундаментальний розв'язок, гармонійні потенціали, тензор деформачій, тензор напружень. 